\title{
Trem2 Variants and Risk of Alzheimer’s Disease
}

Perez Martinez David Andres and Manzano Palomo Maria Sagrario

${ }^{1}$ Neurology Department "Infanta Cristina" University Hospital Parla-Madrid, Spain

"Corresponding author: Perez-Martinez David Andres, Neurology Department "Infanta Cristina” University Hospital Parla-Madrid, Spain, Tel: 913964709; E-mail: daperezm@salud.madrid.org

Received date: Apr 03, 2014, Accepted date: May 19, 2014, Published date: May 26, 2014

Copyright: (C) 2014 David Andres PM, et al. This is an open-access article distributed under the terms of the Creative Commons Attribution License, which permits unrestricted use, distribution, and reproduction in any medium, provided the original author and source are credited

\begin{abstract}
Object: Alzheimer's disease (AD) is the most common form of dementia in the elderly. The genetic basis of lateonset AD (LOAD) is not well known. However, since 1993 the relationship with APOE gene is known, recently it has established a new relationship with the TREM2 gene. This review aims to show the implications of mutations in TREM2 gene in AD.

Background: Mutations in TREM2 have been involved in Nasu-Hakola disease that causes frontotemporal dementia like (FTD-like) phenotype. Recently it has been involved in AD with an odds ratio as strong as previously reported with APOE\&4.

Methods and results: We review relevant papers concerning to TREM2 gene, not only its implication in neurodegenerative disease, but also those focused on Alzheimer's Disease.

Conclusion: There is an increased frequency of rare heterozygous TREM2 variations in AD and FTD, and TREM2 variants may play a role in neurodegenerative diseases in general. However, the relationship between TREM2 gene and neurodegenerative diseases is complex and ambiguous results. Surely the TREM2 variants have great interest in future research of neurodegenerative diseases.
\end{abstract}

Keywords: TREM2; Genetic risk; Alzheimer's disease; Frontotemporal dementia; Nasu-Hakola disease; Late-onset Alzheimer 's disease.

\section{Introduction}

Alzheimer's disease (AD) is a neurodegenerative disorder that is characterized by a slow and progressive loss of cognitive functions. In developed countries it is the most common form of late-onset dementia over 60 years old. The main pathological hallmarks of $\mathrm{AD}$ are extracellular amyloid plaques, intracellular neurofibrillary tangles, and loss of neurons and synapses. These changes result in a cerebral atrophy with cognitive and neuropsychiatric symptoms. The vast majority of cases of Alzheimer's disease are late-onset cases over 60 years. However, there are early forms of AD (EOAD) in which the genetic load is high and associated with mutations in APP, PSEN1, and PSEN2 genes (encoding amyloid precursor protein, presenilin 1, and presenilin 2, respectively). These variants appear to be fully penetrant and result in Alzheimer's disease with an early onset, in most cases before the age of 60 years.

The genetic basis of late onset Alzheimer's Disease (LOAD) are less known and, until recently, only found regarding the gene encoding the $\varepsilon 4$ allele of apolipoprotein $\mathrm{E}$ (ApoE), originally discovered as a risk factor for AD in 1993. APO E is the only susceptibility gene involved in $\mathrm{AD}$ with an odds ratio (OR) 3-4 [1].

Current high-throughput sequencing technologies such as wholeexome and whole-genome sequencing enable the identification of rare genetic causes of disease. Using a whole-exome sequencing approach, homozygous mutations in the gene encoding triggering receptor expressed on myeloid cells 2 (TREM2; 6p21.1) were identified as a cause of behavioral variant frontotemporal dementia (FTD) in 3 consanguineous Turkish families [2].

TREM2 is a receptor of the innate immune system, expressed on the cell membrane of myeloid cells, especially on immature dendritic cells, microglia, and osteoclasts. Homozygous loss-of-function mutations in TREM2 (e.g., p.Q33X) were previously found in patients with NasuHakola disease (polycystic lipomembranous osteodysplasia with sclerosing leukoencephalopathy: PLOSL). This is a rare recessively inherited disease that is characterized by early-onset progressive dementia and bone cysts [3].

\section{TREM2 Gene and its Implication in Neurodegenerative Diseases}

There are a number of pathways that are likely generalized across neurodegenerative diseases, including the mitochondrial dysfunction, ubiquitin-proteasome system and the inflammatory response. There is evidence that inflammation is an early event in the brains of patients with $\mathrm{AD}$ [4]. It has also been noted that the expression of genes associated with inflammation in the brain is increased in aging and that this effect is accentuated in AD. Genome wide association studies have also provided evidence of the importance of inflammation in AD.

TREM2 has an important role in the immune response and its lossof function mutations have been demonstrated to cause a spectrum of dementia-like phenotypes with or without bone cysts $[2,3,5]$. The identification of a rare TREM2 substitution (p.R47H) as a risk factor for $\mathrm{AD}$ suggests that the protein plays an important role in neurodegeneration. TREM2 is known to control two streams of 
signaling, one of these streams regulates phagocytosis. Increased expression of TREM2 on microglia is coupled to enhanced phagocytic pathways and promotes the alternative activation state of microglia, which is thought to be protective [6-8]. The other signaling stream suppresses inflammatory reactivity and involves the repression of cytokine production and secretion. The inflammation response of TREM 2 inhibits macrophage response to ligation of toll-like receptor (TLR) [9], and it negatively regulates TLR-mediated maturation of dendritic cells, type I interferon responses, and the induction of antigen-specific T-cell proliferation [10]. Furthermore, TREM2 stimulation of dendritic cells induces partial activation without any production of pro-inflammatory cytokines [11].

There is growing evidence to support the role of activated microglia pathways in neuro-degeneration. A recent integrated systems approach identified that DAP12 (TYROBP) is one of the key genes. The authors have demonstrated that DAP12 is involved in amyloidbeta turnover and neuronal damage with TREM2, via $\beta$-catenin [12]. Recessive mutations in both TREM2 and DAP12 produce the clinical phenotype of Nasu-Hakola disease [13].

\section{Discussion}

Nasu-Hakola disease and Alzheimer's disease are distinct from each other, and the clinical symptoms of Nasu-Hakola disease (early onset, painful bone cysts, fractures of bones of the limbs, and sclerosing leukoencephalopathy) are incompatible with the diagnosis of $\mathrm{AD}$. Taking into account that, it is possible that rare mutations accounting for a small proportion of cases of common diseases may define a clinical subgroup. It has been reported a homozygous mutation in the $5^{\prime}$ consensus donor splice site in intron 1 of TREM2 in a Lebanese family, leading to early-onset dementia without bone cysts [14].

Furthermore, mutations in TREM2 have been reported in three Turkish probands with frontotemporal dementia-like disease in the absence of bone cysts [15]. In an Italian family, those heterozygous carriers with a loss-of-function mutation in TREM2, have been reported memory deficits [16]. These findings suggest that TREM2 may be crucial for the integrity of cognitive function. The $\mathrm{R} 47 \mathrm{H}$ substitution encoded by rs75932628- $\mathrm{T}$ is located within the extracellular immunoglobulin- like domain of TREM2. The amino acid substitution may result in decreased affinity of TREM2 for its natural ligands and affect its signaling. It has been proposed that TREM2 may represent a proteolytic substrate for $\gamma$-secretase, although the exact cleavage site was not identified [17].

The rare variant p.R47H [18], (rs75932628), has demonstrated a strong association with late onset AD in two different studies [19]. This mutation codifies a protein with a substitution of arginine by histidine in 47 positions, with an allelic prevalence in Iceland $0.63 \%$. This has been replicated in Spain [20] and French [21] population.

In a Belgian population study, the estimated PAF (populationattributable fraction) for TREM2 p.R47H was only $0.55 \%$, very low if you compared with APOE $\varepsilon 4$ one (PAF> 30\%). This study suggests that heterozygotes variants of TREM2 play a rol as risk factors with a moderate penetrance, which is a lack of segregation [5].

The Cache County Study on Memory Health and Aging is a huge population study that began in 1994. It includes 5092 participants that represent $90 \%$ of the whole Cache County inhabitants, in Utah with 65 years old or older. Those with dementia had a blood sample, MRI and cognitive examination, and Alzheimer diagnosis was according NINCDS-ADRDA criteria (National Institute of Neurological and Communicative Disorders and Stroke and the Alzheimer's Disease and Related Disorders Association criteria) [22], included probable cases. In this study $\mathrm{R} 47 \mathrm{H}$ variant was analyzed in late onset $\mathrm{AD}$. The OR was 3.5 with a risk very similar to APOE4 one. However, this variant is very infrequent in general population $(0.004$ vs. 0.20 for APOE ع4) [23].

Also we have information about the TREM2 variants in other neurodegenerative diseases such as amyotrophic lateral sclerosis (ALS). Recently, Cady et al. [24] have published a paper of TREM2 Variant p.R47H as a risk factor for Sporadic ALS. Samples of DNA from 923 individuals with sporadic ALS and 1854 healthy control individuals self-reported as non-Hispanic white were collected from ALS clinics in the United States and genotyped for the p.R47H variant in TREM2. This variant was more common in patients with ALS than in the controls and is therefore a significant risk factor for ALS (odds ratio, 2.40; 95\% CI, 1.29-4.15).

However, in contradiction with previous studies, there is a recently published study of TREM-like receptors with ambiguous results. TREM-Like2 is analogous to the original receptor TREM2 receptor, although this receptor is encoded in another genomic region. Unexpectedly, polymorphisms in this genomic region have been identified to be protective for $\mathrm{AD}$ in a recent paper [25].

In the same direction, a paper published by Miyashita $\mathrm{A}$ et al conducted a study using a well-characterized Japanese sample set, comprising 2,190 late-onset $\mathrm{AD}$ (LOAD) cases and 2,498 controls. They genotyped 10 non-synonymous TREM2 variants with no significant association with LOAD [26].

In conclusion, further insights into the pathophysiology of $\mathrm{AD}$ and, in general, neurodegenerative diseases are needed. Genetics play a main role in a deep knowledge of them and could lead us new therapeutic approaches. TREM2 variants have found a new risk for Alzheimer's disease. Although this variant occurs with less frequency than the ApoE $\varepsilon 4$ allele, it confers a risk of Alzheimer's disease with an effect size that is similar to that of ApoE $\varepsilon 4$. However, TREM2 variants appear to be associated with different phenotypes of neurodegenerative diseases, particularly ALS and FTD. Conversely, recent work with TREM-like 2 variants appears to provide protection against $\mathrm{AD}$. In short, TREM2 variants provide an exciting way to investigate the neurobiological basis of neurodegenerative diseases [27].

\section{References}

1. Mullan M, Crawford F (1993) Genetic and molecular advances in Alzheimer's disease. Trends Neurosci 16: 398-403.

2. Guerreiro R, Bilgic B, Guven G, Brás J, Rohrer J, et al. (2013) Novel compound heterozygous mutation in TREM2 found in a Turkish frontotemporal dementia-like family. Neurobiol Aging 34: 2890.

3. Guerreiro R, Wojtas A, Bras J, Carrasquillo M, Rogaeva E, et al. (2013) TREM2 variants in Alzheimer's disease. N Engl J Med 368: 117-127.

4. Eikelenboom P, van Exel E, Hoozemans JJ, Veerhuis R, Rozemuller AJ, et al. (2010) Neuroinflammation - an early event in both the history and pathogenesis of Alzheimer's disease. Neurodegener Dis 7: 38-41.

5. Cuyvers E, Bettens K, Philtjens S, Van Langenhove T, Gijselinck I, et al. (2014) Investigating the role of rare heterozygous TREM2 variants in Alzheimer's disease and frontotemporal dementia. Neurobiol Aging 35: 726. 
6. Takahashi, Prinz M, Stagi M, Chechneva O, Neumann H (2007) TREM2-transduced myeloid precursors mediate nervous tissue debris clearance and facilitate recovery in an animal model of multiple sclerosis. PLoS Med 4: e124.

7. Frank S, Burbach GJ, Bonin M, Walter M, Streit W, et al. (2008) TREM2 is upregulated in amyloid plaque-associated microglia in aged APP23 transgenic mice. Glia 56: 1438-1447.

8. Piccio L, Buonsanti C, Mariani M, Cella M, Gilfillan S, et al. (2007) Blockade of TREM-2 exacerbates experimental autoimmune encephalomyelitis. Eur J Immunol 37: 1290-1301.

9. Hamerman JA, Jarjoura JR, Humphrey MB, Nakamura MC, Seaman WE et al. (2006) Cutting edge: inhibition of TLR and FcR responses in macrophages by triggering receptor expressed on myeloid cells (TREM)-2 and DAP12. J Immunol 177: 2051-2055.

10. Ito H, Hamerman JA (2012) TREM-2, triggering receptor expressed on myeloid cell-2, negatively regulates TLR responses in dendritic cells. Eur J Immunol 42: 176-185.

11. Bouchon A, Hernandez-Munain C, Cella M, Collona M (2001).A DAP12-mediated pathway regulates expression of CC chemokine receptor 7 and maturation of human dendritic cells. J Exp Med 194:1111-1122

12. Otero K, Turnbull IR, Poliani PL, Vermi W, Cerutti E, et al. (2009) Macrophage colony-stimulating factor induces the proliferation and survival of macrophages via a pathway involving DAP12 and betacatenin. Nat Immunol 10: 734-743.

13. Paloneva J, Kestilä M, Wu J, Salminen A, Böhling T, et al. (2000) Loss-offunction mutations in TYROBP (DAP12) result in a presenile dementia with bone cysts. Nat Genet 25: 357-361.

14. Chouery E, Delague V, Bergougnoux A, Koussa S, Serre JL, et al. (2008) Mutations in TREM2 lead to pure early-onset dementia without bone cysts. Hum Mutat 29: E194-204.

15. Guerreiro RJ, Lohmann E, Brás JM, Gibbs JR, Rohrer JD, et al. (2013) Using exome sequencing to reveal mutations in TREM2 presenting as a frontotemporal dementia-like syndrome without bone involvement. JAMA Neurol 70: 78-84.

16. Montalbetti L, Ratti MT, Greco B, Aprile C, Mogile A et al. (2005). Neuropsychological tests and functional nuclear neuroimaging provide evidence of subclinical impairment in Nasu-Hakola disease heterozygotes. Funct Neurol. 20:71-75
17. Wunderlich P, Glebov K, Kemmerling N, Tien NT, Neumann H et al (2013). Sequential proteolytic processing of the triggering receptor expressed on myeloid cells-2 (TREM2) protein by ectodomain shedding and gamma-secretase-dependent intramembranous cleavage. J BiolChem 288:33027-33036

18. Ruiz A, Dols-Icardo O, Bullido MJ, Pastor P, Rodríguez-Rodríguez E, et al. (2014) Assessing the role of the TREM2 p.R47H variant as a risk factor for Alzheimer's disease and frontotemporal dementia. Neurobiol Aging 35: 444.

19. Jonsson T, Stefansson H, Steinberg S, Jonsdottir I, Jonsson PV, et al (2013) Variant of TREM2 associated with the risk of Alzheimer's disease. N Engl J Med 368: 107-116.

20. Benitez BA, Cooper B, Pastor P, Jin SC, Lorenzo E, et al. (2013) TREM2 is associated with the risk of Alzheimer's disease in Spanish population. Neurobiol Aging 34: 1711.

21. Pottier C, Wallon D, Rousseau S, Rovelet-Lecrux A, Richard AC, et al. (2013) TREM2 R47H variant as a risk factor for early-onset Alzheimer's disease. J Alzheimers Dis 35: 45-49.

22. McKhann G, Drachman D, Folstein M, Katzman R, Price D, et al. (1984) Clinical diagnosis of Alzheimer's disease: report of the NINCDS-ADRDA Work Group under the auspices of Department of Health and Human Services Task Force on Alzheimer's Disease. Neurology 34: 939-944.

23. Gonzalez Murcia JD, Schmutz C, Munger C, Perkes A, Gustin A, et al. (2013) Assessment of TREM2 rs75932628 association with Alzheimer's disease in a population-based sample: the Cache County Study. Neurobiol Aging 34: 2889.

24. Cady J, Koval ED, Benitez BA, Zaidman C, Jockel-Balsarotti J, et al. (2014) TREM2 variant p.R47H as a risk factor for sporadic amyotrophic lateral sclerosis. JAMA Neurol 71: 449-453.

25. Benitez BA, Jin SC, Guerreiro R, Graham R, Lord J, et al. (2014) Missense variant in TREML2 protects against Alzheimer's disease. Neurobiol Aging 35: 1510.

26. Miyashita A, Wen Y, Kitamura N, Matsubara E, Kawarabayashi T, et al. (2014) Lack of Genetic Association Between TREM2 and Late-Onset Alzheimer's Disease in a Japanese Population. J Alzheimers Dis .

27. Jones BM, Bhattacharjee S, Dua P, Hill JM, Zhao Y, et al. (2014) Regulating amyloidogenesis through the natural triggering receptor expressed in myeloid/microglial cells 2 (TREM2). Front Cell Neurosci 8: 94. 